\title{
The Impact of Land Use Change on the Ecosystem Metabolome of a Forested Watershed
}

Claudia M. Boot" Allison HaAse', Timothy Fegel, Ed Hall', Charles Rhodes', Timothy CovinO'

Colorado State University, Fort Collins, CO 80523, USA

(*correspondence: claudia.boot@ colostate.edu)

U.S. Forest Service, Rocky Mountain Research Station, Fort Collins, CO 80526, USA

Land management strategies have a substantial impact on the composition and reactivity of dissolved organic matter (DOM) exported from forested watersheds. We studied this relationship by collecting lateral subsurface flow from adjacent old growth and second-growth conifer stands in the Fraser Experimental Forest, CO. The quantity of DOM exported from the second-growth stand was a fraction of that from the old-growth stand, but its reactivity was an order of magnitude greater. We used mass spectrometry (MS) based ecosystem metabolomics in order to link the characteristics of the DOM pool with the effects of forest management on DOM composition and lability.

We used liquid chromatography inline with quadrupole time of flight tandem MS to conduct an untargeted analysis of the small molecule profiles of DOM from subsurface flow samples. The DOM MS fragmentation spectra were analyzed using the Global Natural Product Social Molecular Networking (GNPS) platform, which indicated specific groups of molecules with common structural features were responsive to management strategy. Responsive molecules were selected for further and on-going analyses including molecular formula (MF) determination, MF-based stoichiometric characteristics, as well as in silico prediction of their hierarchical chemical taxonomy.

High-resolution characterization of the ecosystem metabolome has the potential to descriptively link specific molecules and compound classes to ecosystem traits and function. 